Condensed Matter Physics, 1998, Vol. 1, No. 3(15), p. 569-574

\title{
The canonical $u-v$ Bogolubov transformation in a weakly relativistic statistical theory of a charged particle system
}

\author{
L.F.Blazhyjevskii, Yu.S.Krynytskyi \\ Ivan Franko Lviv State University, Department for Theoretical Physics, \\ 12 Drahomanov St., UA-290005 Lviv, Ukraine
}

Received May 6, 1998

\begin{abstract}
A weakly relativistic statistical system of charged particles and an electromagnetic field is considered. The effective Hamiltonian of the system is obtained by the canonical Bogolubov transformation. The screening of the relativistic direct interaction of particles and renormalization of the photon energy spectrum are shown.
\end{abstract}

Key words: weakly relativistic statistical theory, Bogolubov transformation

PACS: $05.20 .-y, 03.30 .+p$

It is well known that there is a wide range of physical phenomena for the description of which it is necessary to account relativistic effects. Generally speaking, the most suitable theory of such phenomena must be the field theory. Nevertheless, for a long time an alternative description of relativistic systems on the basis of direct interaction theories is being developed. These are the theories where interactions between the particles are described by some functions (or functionals) which are depended only on particle variables. Apparently, one of the first such theories is the Wheeler-Feynman electrodynamics. Another trend of developing direct interaction theories is based on deriving the main expressions for series by power of $c^{-1}$ ( $c$ is the velocity of light in the vacuum). It is sufficient to account only a few first terms of the series because the relativistic effects are often small. And, moreover, in the lowest approximations it is possible to fully neglect the field degrees of freedom (for electromagnetic and gravitational interactions they appear only in $c^{-3}$ and $c^{-5}$ approximations, accordingly). In higher approximations it is possible to account the influence of the field degrees of freedom with the help of introduction of higher derivatives into the theory. An important contribution to developing such theories was made by Professor Gaida and his followers. Separately proceeding from such general principles of the relativistic direct interaction 
theories like causality, Poincaré invariance and separability of interactions, Prof. Gaida found the general appearance of Lagrangians and Hamiltonians on the basis of which both the classical and quantum mechanics are formulated. A series of results are given in review [1].

Successful development of the relativistic direct interaction theories to a great extent stimulated the carrying out of such an investigation in statistical physics. However, it is shown that formulating the post-Newtonian (weakly relativistic) statistical mechanics on the basis of canonical alignment the post-Newtonian classical mechanics is not always possible. It is connected with the necessity to take into account the existence of thermodynamic limit $(N \rightarrow \infty, V \rightarrow \infty, N / V \rightarrow$ const $)$ when Lagrange variables transform to Hamilton ones (that process is always approximate). As it is shown in [2], any finite developing to series by power $c^{-1}$ does not coordinate with thermodynamic limit for long-range interaction systems. Therefore, it is necessary to take into account all the terms of series by power $c^{-1}$ and the Hamilton function will intricately depend on parameter $c^{-1}$. The Green functions and Liouville distribution will be also complicated because they are constructed from the Hamilton function. For post-Newtonian systems of charged particles this Hamiltonian was firstly obtained in [3] from the Darwin Lagrangian. In the present paper we show that the same result can be obtained on the basis of the canonical $u-v$ Bogolubov transformation which is widely used in the theories of superconductivity and superfluidity.

Let us consider the system of $N$ charged particles and the electromagnetic field that is created by the particles. It is convenient to use the Coulomb gauge of electromagnetic potentials. At the same time the Hamiltonian of the system can be described by the following expressions, including terms that are proportional to $1 / c^{3}$ :

$$
\begin{aligned}
& H=H_{f}+H_{p}+H^{\prime}+H^{\prime \prime} \\
& H_{f}=\sum_{k} \sum_{\lambda=1,2} \hbar c k b_{\boldsymbol{k}, \lambda}^{+} b_{\boldsymbol{k}, \lambda} \\
& H_{p}=\sum_{j=1}^{N}\left\{\frac{p_{j}^{2}}{2 m}-\frac{p_{j}^{4}}{8 m^{3} c^{2}}\right\}+U_{c} \\
& H^{\prime}=-\sum_{j=1}^{N} \frac{e}{m c}\left(\boldsymbol{p}_{j}, \boldsymbol{A}\left(\boldsymbol{r}_{j}\right)\right)\left[1-\left(\frac{p_{j}}{m c}\right)^{2}\right], \quad H^{\prime \prime}=\frac{e^{2}}{2 m c^{2}} \sum_{j=1}^{N} A^{2}\left(\boldsymbol{r}_{j}\right), \\
& \boldsymbol{A}\left(\boldsymbol{r}_{j}\right)=\sum_{\boldsymbol{k}} \boldsymbol{A}_{\boldsymbol{k}} e^{i \boldsymbol{k r}_{j}}, \quad \boldsymbol{A}_{\boldsymbol{k}}=\left(\frac{2 \pi \hbar c}{V k}\right)^{\frac{1}{2}} \sum_{\lambda=1,2}\left(\boldsymbol{e}_{\lambda}(k) b_{\boldsymbol{k}, \lambda}^{+}+\boldsymbol{e}_{\lambda}(-k) b_{-\boldsymbol{k}, \lambda}\right),
\end{aligned}
$$

where $b_{\boldsymbol{k}, \lambda}^{+}, b_{\boldsymbol{k}, \lambda}$ are photon operators of creation and annihilation, $\boldsymbol{e}_{\lambda}(k)$ are normalized polarization vectors, $U_{c}$ is the Coulomb interaction energy, $V$ is the volume of the system. Next calculations are carried out by the use of a random phase approximation, when only interactions between particles with equal pass impulse are taken into account. In this case $\sum_{j} A^{2}\left(\boldsymbol{r}_{j}\right) \simeq N \sum_{\boldsymbol{k}} A_{\boldsymbol{k}}^{2}$ and $H^{\prime \prime}$ can be united 
with the Hamiltonian of free electromagnetic field $H_{f}$. After the accounting of ortogonalization of polarization vectors $\left(\left(\boldsymbol{e}_{\lambda}(k), \boldsymbol{e}_{\lambda^{\prime}}(k)\right)=\delta_{\lambda, \lambda^{\prime}}\right)$ we find

$$
\begin{aligned}
& H_{f}+H^{\prime \prime}=\sum_{\boldsymbol{k}, \lambda} T_{\boldsymbol{k}, \lambda} b_{\boldsymbol{k}, \lambda}^{+} b_{\boldsymbol{k}, \lambda}+ \\
& +\frac{1}{2} \sum_{\boldsymbol{k}, \lambda} \Phi_{\boldsymbol{k}}\left(b_{\boldsymbol{k}, \lambda}^{+} b_{-\boldsymbol{k}, \lambda}^{+}+b_{-\boldsymbol{k}, \lambda} b_{\boldsymbol{k}, \lambda}\right)+\frac{1}{2} \sum_{\boldsymbol{k}, \lambda} \Phi_{\boldsymbol{k}},
\end{aligned}
$$

where

$$
T_{\boldsymbol{k}}=\hbar c k\left(1+\frac{\omega_{0}^{2}}{2 c^{2} k^{2}}\right), \quad \Phi_{\boldsymbol{k}}=\frac{\omega_{0}^{2}}{2 c k}, \quad \omega_{0}^{2}=\frac{4 \pi N e^{2}}{V m} .
$$

It is well known that such quadratic forms can be diagonalized by the canonical Bogolubov transformation $[4,5]$. In our case it is described by the formulas:

$$
b_{\boldsymbol{k}, \lambda}=u_{\boldsymbol{k}} \alpha_{\boldsymbol{k}, \lambda}+v_{\boldsymbol{k}} \alpha_{-\boldsymbol{k}, \lambda}^{+}, \quad b_{\boldsymbol{k}, \lambda}^{+}=u_{\boldsymbol{k}} \alpha_{\boldsymbol{k}, \lambda}^{+}+v_{\boldsymbol{k}} \alpha_{-\boldsymbol{k}, \lambda} .
$$

After the necessary calculations we find

$$
H_{f}+H^{\prime \prime}=\sum_{\boldsymbol{k}, \lambda} E_{\boldsymbol{k}} \alpha_{\boldsymbol{k}, \lambda}^{+} \alpha_{\boldsymbol{k}, \lambda}+K, \quad H^{\prime}=-\sum_{\boldsymbol{k}, \lambda}\left(\gamma_{\boldsymbol{k}, \lambda} \alpha_{\boldsymbol{k}, \lambda}^{+}+\gamma_{-\boldsymbol{k}, \lambda} \alpha_{\boldsymbol{k}, \lambda}\right) .
$$

Here the following expressions are accepted:

$$
\begin{aligned}
& E_{k}=\sqrt{T_{k}^{2}-\Phi_{k}^{2}}, \quad K=\frac{1}{2} \sum_{k, \lambda}\left(E_{k}-T_{\boldsymbol{k}}+\Phi_{\boldsymbol{k}}\right) \\
& \gamma_{\boldsymbol{k}, \lambda}=\frac{e}{m c}\left(\frac{2 \pi \hbar c}{V k}\right)^{\frac{1}{2}}\left(u_{\boldsymbol{k}}+v_{\boldsymbol{k}}\right) \sum_{j} e^{i \boldsymbol{k r}_{j}}\left[1-\left(\frac{p_{j}}{m c}\right)^{2}\right]\left(\boldsymbol{p}_{j}, \boldsymbol{e}_{\lambda}(k)\right), \\
& u_{\boldsymbol{k}}=\sqrt{T_{\boldsymbol{k}}+E_{\boldsymbol{k}}} / \sqrt{2 E_{\boldsymbol{k}}}, \quad v_{\boldsymbol{k}}=-\sqrt{T_{\boldsymbol{k}}-E_{\boldsymbol{k}}} / \sqrt{2 E_{\boldsymbol{k}}} .
\end{aligned}
$$

Let us consider only the classical statistical system of charged particles. Then in (4) we can except linear for $\alpha_{\boldsymbol{k}, \lambda}^{+}, \alpha_{\boldsymbol{k}, \lambda}$ terms by the translation transform. We include new operators (analogous to $[5]$ ):

$$
\eta_{\boldsymbol{k}, \lambda}=\alpha_{\boldsymbol{k}, \lambda}+\gamma_{\boldsymbol{k}, \lambda} / E_{\boldsymbol{k}}, \quad \eta_{\boldsymbol{k}, \lambda}^{+}=\alpha_{\boldsymbol{k}, \lambda}^{+}+\gamma_{-\boldsymbol{k}, \lambda} / E_{\boldsymbol{k}},
$$

that do not break the commutation alignments of the Bose statistics. After transforming to new variables in (4) we find:

$$
H_{f}+H^{\prime \prime}+H^{\prime}=K+\sum_{\boldsymbol{k}, \lambda} E_{\boldsymbol{k}} \eta_{\boldsymbol{k}, \lambda}^{+} \eta_{\boldsymbol{k}, \lambda}-\sum_{\boldsymbol{k}, \lambda} \gamma_{\boldsymbol{k}, \lambda} \gamma_{-\boldsymbol{k}, \lambda} / E_{\boldsymbol{k}}
$$

The last term describes relativistic corrections to the interaction. After taking into account expressions (3), (5) and the formula

$$
\sum_{\lambda} e_{\lambda}^{\mu}(k) e_{\lambda}^{\nu}(-k)=\delta_{\mu \nu}-k_{\mu} k_{\nu} / k^{2}, \quad \mu, \nu=x, y, z,
$$


we can transform the last term in (6) into the following form:

$$
\begin{aligned}
& \frac{1}{2} \sum_{j, l}\left(1-\frac{p_{j}^{2}}{m^{2} c^{2}}\right)\left(1-\frac{p_{l}^{2}}{m^{2} c^{2}}\right) \mathcal{H}_{j l}, \\
& \mathcal{H}_{j l}=-\frac{1}{m^{2} c^{2}} \sum_{\boldsymbol{k}} \frac{4 \pi e^{2}}{V k^{2}}\left[1+\frac{\omega_{0}^{2}}{k^{2} c^{2}}\right]^{-1}\left[\left(\boldsymbol{p}_{j}, \boldsymbol{p}_{l}\right)-\left(\boldsymbol{k}, \boldsymbol{p}_{j}\right)\left(\boldsymbol{k}, \boldsymbol{p}_{l}\right) / k^{2}\right] .
\end{aligned}
$$

Since $\mathcal{H}_{j l} \sim 1 / c^{2}$, we can neglect the terms $p^{2} / m^{2} c^{2}$ in (7). Moreover, it is necessary to carry out renormalization of the particle mass. For that it is sufficient to withdraw from $(7)$ the terms of self-action (the terms with $j=l$ ) that do not depend on $\omega_{0}^{2}$. The rest of self-action terms are united with the Hamiltonian of the particles and it leads to the appearance of effective mass $m^{*}=m\left(1-2 e^{2} \omega_{0} / 3 m c^{3}\right)$ (see [3]).

After the analysis of the obtained results we can see that Hamiltonian (1) in the post-Newtonian approximation can be written in the form of a sum of three terms. They are the Hamiltonian of field $\mathcal{H}_{f}$, the energy of zero oscillations $K$ and the Hamiltonian of particles $\mathcal{H}_{p}$ :

$$
\begin{aligned}
\mathcal{H}_{f} & =\sum_{\boldsymbol{k}, \lambda} E_{\boldsymbol{k}} \eta_{\boldsymbol{k}, \lambda}^{+} \eta_{\boldsymbol{k}, \lambda}, \quad E_{\boldsymbol{k}}=\hbar \sqrt{c^{2} k^{2}+\omega_{0}^{2}}, \\
K & =\frac{1}{2} \sum_{\boldsymbol{k}, \lambda}\left(E_{\boldsymbol{k}}-\hbar c k\right), \\
\mathcal{H}_{p} & =\sum_{j}\left(\frac{p_{j}^{2}}{2 m^{*}}-\frac{p_{j}^{4}}{8 m^{3}} c^{2}\right)+U_{c}+\frac{1}{2} \sum_{j \neq l} \mathcal{H}_{j l},
\end{aligned}
$$

Let us make several comments. Expression (9) can be interpreted as an effective Hamiltonian of the electromagnetic field. Since $E_{\boldsymbol{k}}$ is real, then it is possible to describe the photons as quasiparticles that correspond to elementary excitements of the particles and the field, and $E_{k}$ is the energy of the photon-quasiparticle. The difference of $E_{k}$ from $\hbar c k$ determines the changing of the radiation spectral distribution. As it is shown in [6], the corresponding correction to the radiation energy of an absolutely black body can be described by the formula $-\pi e^{2} \theta^{2} / 3 \hbar m c^{3}$ ( $\theta$ is statistical temperature).

The energy of zero oscillations $K$ has a divergent value. But this infinite constant does not influence the thermodynamic and kinetic properties of the system because we can get rid of $K$ by renormalization. Actually, after introducing into (10) the limit impulse $\hbar k_{0}$ we shall obtain:

$$
K=N \frac{\hbar e^{2} k_{0}^{2}}{2 \pi m c}-\frac{N^{2}}{V} \frac{\hbar e^{4}}{m^{2} c^{3}}\left[\ln \left(2 \frac{k_{0} c}{\omega_{0}}\right)-\frac{1}{4}\right] .
$$

The first term can be neglected because it describes the changing of the vacuum state energy of the field that is connected with the interaction of the particles and 
the field (if we use a normal regularization of the field operator in (2), this term will not appear). The second term disappears in consequence of mass renormalization. Actually, a bare mass is included into (12) and for its transition to a physical mass it is necessary to substitute $m+\delta m$ for $m$ where $\delta m$ is an electromagnetic mass. Since $\delta m \sim \ln \left(\hbar k_{0} / m c\right)$, then the second term in (12) is proportional to $\delta m /(m+\delta m)^{2}$ and disappears if $\delta m \rightarrow \infty$.

$\mathcal{H}_{p}$ has the substance of an effective particles Hamiltonian. Formulas (8), (11) coincide with the results that were obtained in [3] from the Darwin Lagrangian by the Legendre transformation. Transformation to canonical variables is connected with summing some infinite series by power $c^{-2}$. Within the framework of a field approach the similar results were obtained in [7]. As we can see from (8), (11), the relativistic interaction between the particles is screened at the distance $c / \omega_{0}$. The appearance of screening and effective particle mass $m^{*}$ is the consequence of manyparticle character of a long-range electromagnetic interaction in canonical variables (note that for the classical Newtonian mechanics in the case of $N>3$ in Hamilton variables the interaction does not reduce to the sum of pair interactions (see [1])). In the random phase approximation many-particle interactions are substituted for effective two-particle interactions that are described by a screened potential. From the formal point of view the appearance of screening is connected with taking into account term $H^{\prime \prime}$ in (1). The obtained formulas do not contradict the results of mechanics. In classical mechanics $N$ and $V$ are not connected variables. Therefore, after $V \rightarrow \infty$ and $\omega_{0} \rightarrow 0$ from formulas (8), (11), we shall have the classical expression that is analogous to a weakly relativistic Breit Hamiltonian (see [8]).

Using an effective Hamiltonian (11) for the calculation of thermodynamic functions $\delta F_{r}$ does not lead to divergences. For example, the relativistic correction to the free energy of the classical system of charged particles in the random phase approximation is determined by the sum of ring contributions $\left\langle\mathcal{H}_{12} \mathcal{H}_{23} \ldots \mathcal{H}_{n 1}\right\rangle$ (symbol $\langle\ldots\rangle$ signifies the averaging by the distribution function of non-interactive particles). If $\mathcal{H}_{j l}$ is the Breit Hamiltonian, the sum of those contributions can be represented by the divergent series

$$
\delta F_{r}=-\frac{\theta}{2} \sum_{k} \sum_{n \geqslant 2} \frac{1}{n}\left(\frac{\omega_{0}^{2}}{c^{2} k^{2}}\right)^{n} .
$$

After performing the same calculations with Hamiltonians (8), (11), we shall obtain the series:

$$
\delta F_{r}=-\frac{\theta}{2} \sum_{k} \sum_{n \geqslant 2} \frac{1}{n}\left(\frac{\omega_{0}^{2}}{c^{2} k^{2}+\omega_{0}^{2}}\right)^{n},
$$

that is obviously convergent.

\section{References}

1. Gaida R. P. Quasirelativistic systems of interacting particles. // Fiz. Elem. Chast. \& Atom. Yadra, 1982, vol. 13, No 2, p. 427-493 (in Russian). 
2. Blazhievsky L. F., Hil' H. B., Semak S. S. Some problems of the construction of weakly relativistic statistical mechanics // Journal of Physical Studies, 1996, vol. 1, No 1, p. 1-11 (in Ukrainian).

3. Blazhievsky L. F. On statistical thermodynamics of quasi-relativistic charged particle system // Ukr. Fiz. Zhurn., 1975, vol. 20, No 8, p. 1273-1281 (in Russian).

4. Bogolubov N. N. Selected Works, vol. 2. Kiev, Naukova Dumka, 1970, 522 p. (in Russian).

5. Bogolubov N. N., Bogolubov N. N. (jr.) Introduction to Quantum Statistical Mechanics. Moscow, Nauka, 1984, 384 p. (in Russian).

6. Blazhyjevskii L. F. Field aspects of the method of collective variables for the system of charged particles // Condensed Matter Physics, 1995, No 6, p. 23-34.

7. Jones R. D., Pytte A. Hamiltonian of a slightly relativistic plasma // Phys. Rev. D, 1980, vol. 23, No 2, p. 269-272.

8. Landau L. D., Lifshitz E. M. Quantum Electrodynamics. Course of Theoretical Physics, vol. 4. Moscow, Nauka, 1989, 728 p. (in Russian).

\section{Канонічне $u-v$ перетворення Боголюбова у слабкорелятивістичній теорії систем заряджених частинок}

\section{Л.Ф.Блажиєвський, Ю.С.Криницький}

Львівський державний уріверситет ім. І. Франка, кафедра теоретичної фізики, 290005 м. Львів, вул. Драгоманова, 12

Отримано 6 травня 1998 р.

Розглядається статистична слабкорелятивістична система заряджених частинок і електромагнетного поля. Канонічним перетворенням Боголюбова отримано ефективний гамільтоніян системи. Виявлено екранування релятивістичної прямої взаємодії та перенормування енерґетичного спектру фотонів.

Ключові слова: слабкорелятивістична теорія, перетворення Боголюбова

PACS: $05.20 .-y, 03.30 .+p$ 